
\title{
3 Research Square \\ Bone Response in vivo of Ti-45Zr Alloy as Dental Implant Material
}

Pinghua Ou

Central South University

Taomei Zhang

Central South University

Jianying Wang

Central South University

Cui Li

Central South University

Chunsheng Shao

Central South University

Jianming Ruan ( $\square$ jianming@csu.edu.cn )

Central South University https://orcid.org/0000-0002-0472-7982

\section{Research}

Keywords: dental implant material, biocompatibility, osseointegration ability, immune response

Posted Date: July 7th, 2021

DOI: https://doi.org/10.21203/rs.3.rs-673634/v1

License: (c) (i) This work is licensed under a Creative Commons Attribution 4.0 International License.

Read Full License 


\section{Abstract}

\section{Background}

Ti-Zr alloys have gain more attention as a new metallic biomaterial. using as implants both for orthopedics and dentistry. More recently, our group found promising results for Ti-45Zr alloy, while presenting a low elastic modulusand, a pronounced excellent mechanic character and excellent cell compatibility in vitro. However, the biocompatibility and the potential to promote osteogenesis remains unclear.

\section{Methods}

In this study, the biocompatibility, osteointegration ability, and immune response effects of Ti-45Zr alloy were systematicly investigated in vivo. The biocompatibility of Ti-45Zr alloy was evaluated by hemolysis test and in vivo implantation test in New Zealand white rabbits. The bone integration ability of Ti-45Zr alloy with tissue interface was also investigated by hard tissue section staining and biomechanical experiments. At the same time, the effects of Ti-45Zr alloy on the immune response of Sprague Dawley rats were studied through serum biochemical detection and immunohistochemistry (IHC) staining of hard tissue sections. Interleukin-6 (IL-6) and Interleukin-10 (IL-10) was detected by ELISA.

Results

Results showed that the alloy had good blood compatibility and no body side effects.

After implantation in vivo, The serum concentrations of anti-inflammatory and proinflammatory factors reached the highest values at the 9th day, and the inflammation level around the implant hole returned well with the prolongation of the implantation time. On the 14th day, new bone formation was observed around the three materials. The Ti-45Zr alloy was conducive to the polarisation balance of the macrophages.

\section{Conclusion}

The Ti-45Zr alloy showed exccelent biocompatibility and good osteointegration ability, and it is beneficial to the polarization balance of macrophages.It confirmed that the Ti-45Z $\mathrm{r}$ alloy can be used as a dental implant material.

\section{Introduction}

Dental defects are common oral diseases. They can affect patients' chewing function, leading to alveolar bone and masticatory muscle atrophies, facial collapse, and other problems. Complete dentition loss will lead to patients suffering from malnutrition syndrome, which will affect life quality. Thus, tooth loss repair is particularly important in oral treatment. Dental implant prosthesis became a popular treatment for those defects, due to a high success rate, good predictability, and fewer complications. At present, the 
main reasons for oral implant repair failure in clinical applications include the absence of osseointegration between implant material and bone tissue, peri-implantitis, mechanical fracture, and implant loosening. Therefore, high strength, low elasticity modulus, corrosion resistance, biocompatibility, and osteointegration are key characteristics of implant materials for dental and orthopedic applications.

Biocompatibility refers to the material's normal function in the body. The ideal biomedical implant material must be non-toxic and not cause an allergic reaction in the body. At the same time, the material must not induce a body's immune rejection reaction and coexist harmlessly for a long time. Implant surgery success mainly depends on the body's response to the implant material, so it is essential to evaluate the material's biocompatibility [1]. Frequently, in vivo biocompatibility studies are evaluated in animal implantation experiments. The use of animal models is an important step for dental implant materials testing before clinical application in humans. Implant experiments in animals can mimic the internal environment to determine the optimal interface between the bone and the implanted material. Animal implantation tests are a pre-clinical test for biocompatible materials, and mainly detect toxic reactions, immune responses, and osteointegration ability in vivo.

In this study, a novel dental implant material, the Ti-45Zr alloy which has good biomechanical properties and excellent cytocompatibility [2], was studied in animal implantation experiments. Toxic and side effects, immune responses, and bone integration were systematically studied to provide the theoretical basis for Ti-45Zr alloy clinical application.

\section{Materials And Methods}

\subsection{Sample preparation}

Ti-45Zr alloy was provided by the Powder Metallurgy Research Institute of Central South University. The Ti-45Zr alloy was prepared into a cylinder ( $\Phi 4 \mathrm{~mm} \times 6 \mathrm{~mm}, \Phi 1.5 \mathrm{~mm} \times 4 \mathrm{~mm}$ ) and a circular metal sheet (diameter of $10 \mathrm{~mm}$ and thickness of $1 \mathrm{~mm}$ ) by a mechanical cutting method. Then, it was mechanically polished with \#100, \#200, \#800, and \#1200 silicon carbide sandpaper, and cleaned by ultrasonic wave with acetone, ethanol, and deionized water for $15 \mathrm{~min}$ each. After cleaning, samples were sterilized by a vacuum high-temperature disinfection machine (Vacuklav24B/L+, Germany). After drying, samples were aseptically packaged. Cylindrical samples were used for the animal implantation experiment, and round metal pieces were used for the hemolysis experiment.

\subsection{Hemolysis test}

Blood from healthy volunteers $(20 \mathrm{~mL})$ was extracted and placed in a centrifuge tube $(50 \mathrm{~mL})$, and $1 \mathrm{~mL}$ of freshly prepared $2 \%$ potassium oxalate normal saline solution was added for anticoagulation. The anticoagulant human blood and normal saline (0.9\%) were mixed in a 4:5 ratio (diluted blood) and stored at $4{ }^{\circ} \mathrm{C}$ for later use. After polishing and ultrasonic cleaning, the metal sheet sample was placed in a 15 $\mathrm{mL}$ centrifuge tube and $10 \mathrm{~mL}$ normal saline (0.9\%) was added (experimental group). The negative control group consisted of $10 \mathrm{~mL}$ of normal saline (0.9\%) without a metal sheet, and the positive control 
group of $10 \mathrm{~mL}$ distilled water without a metal sheet. Centrifuge tubes were placed in a $37^{\circ} \mathrm{C}$ thermostatic water tank for preheating for $30 \mathrm{~min}$. Diluted blood $(0.2 \mathrm{~mL})$ was added to each centrifuge tube and kept for $60 \mathrm{~min}$ in a $37^{\circ} \mathrm{C}$ thermostatic water tank. Then, each centrifuge tube was removed and centrifuged (3000 rpm/5 min). The supernatant was placed in a spectrophotometer and the absorbance was measured at $545 \mathrm{~nm}$. The experiment was repeated 3 times for each group.

\subsection{Immune responses in vivo 2.3.1. Surgical method}

Sprague Dawley (SD) rats (12 weeks old) were used as animal models. Before implantation, $10 \%$ chloral hydrate solution $(0.3 \mathrm{~mL} / 100 \mathrm{~g})$ was injected intraperitoneally for anesthesia. After the rats entered the anesthetic state, hair removal and disinfection were performed in the operative area. The femur middle segment surface skin was cut open, subcutaneous tissue and muscle were separated, and the medial femur was exposed. Then, the periosteum was removed with a periosteum stripper to fully expose the visual field. A cylindrical hole $(\Phi 1.5 \mathrm{~mm} \times 4 \mathrm{~mm}$ ) was prepared on the femur right middle segment vertical surface. Next, the implant material was placed into the hole, the wound was washed with normal saline, and the incision was layered sutured (Fig. 1). On the first day after surgery, all rats received a 0.3 $\mathrm{ml}$ cefpiamine $(0.02 \mathrm{~g} / \mathrm{ml})$ intramuscular injection to prevent infection. At postoperative 3,9 , and 14 days, rats were sacrificed by chloral hydrate anesthetic injection. The wound healing was observed and rats' femurs were dissected, removed, and fixed in $4 \%$ paraformaldehyde.

\subsubsection{Enzyme-linked immunosorbent assay (ELISA)}

Fresh blood was collected from the experimental and the control groups at three time points (days 3, 9, and 14) and serum was collected for ELISA. Rat interleukin-6 (IL-6) and interleukin-10 (IL-10) kits (Jiangsu Jingmei) were used according to the manufacture's instructions. The serum Optical Density (OD) was measured at $450 \mathrm{~nm}$. A standard curve was prepared to calculate the concentration of inflammatory factors. Then, the levels of IL- 6 and IL-10 in serum were analyzed.

\subsubsection{Hard tissue sections hematoxylin-eosin (HE) staining}

After the implant material removal, femur samples were decalcified, dehydrated, and embedded. Fixed bone tissue samples were immersed in 15\% EDTA solution for continuous decalcification (30 days). The solution was changed weekly. Then, samples were dehydrated (automatic dehydrator), embedded in paraffin, and sectioned (about $7 \mu \mathrm{m}$ thick). Sections were stained with $\mathrm{HE}$, and images were collected using an Olympus BX50 biological microscope.

\subsubsection{Immunohistochemical staining}

Macrophage surface markers immunohistochemical staining was performed on the decalcified sections and images were collected. Each section was observed at 100x magnification, and 5 field images were randomly selected at $400 x$ magnification. The image analysis system Image-Pro Plus 6.0 was used to 
measure the optical density (IOD) and image's dyed area. The mean density of each image was calculated and the average value of 5 images was considered as the experimental result. Additionally, the number of cells with antigen $68(\mathrm{CD} 68)$ positive $\left(\mathrm{CD} 68^{+}\right)$, nitric oxide synthase (iNOS) positive $\left(\mathrm{iNOS}^{+}\right)$, and arginase-1 (Arg1) positive ( $\mathrm{Arg}^{+}$) differentiation around the implant cluster was counted at 400 fold. By calculating the ratio of $\mathrm{iNOS}^{+}$and $\mathrm{Arg}^{+}$cells to $\mathrm{CD} 68^{+}$cells, the $\mathrm{M} 1$ and $\mathrm{M} 2$ types polarization ratio of macrophages was evaluated. The primary antibodies were CD68, INOS2 and Arg1. At 3, 9, and 14 days, samples were stained with CD68, INOS2, and Arg1 immunohistochemistry to analyze the early postimplantation inflammatory response and macrophages' biological behavior.

\subsection{In vivo osseointegration \\ 2.4.1. Surgical method}

New Zealand male white rabbits (NZW, $12 \mathrm{w}, 2.5 \pm 0.5 \mathrm{~kg}$ ), purchased from the Experimental Animal Center of Central South University, were selected to evaluate the alloy's biosecurity in vivo. After two weeks of domestication, feeding, and environment adaptation, a $3 \%$ pentobarbital solution $(2 \mathrm{~mL} / \mathrm{kg})$ was intravenously injected into the ear margin to put animals under general anesthesia. Then, $2 \%$ lidocaine was injected into the surgical site for local infiltration into the periosteum anesthesia. A longitudinal incision $(2 \mathrm{~cm}$ ) was made on the knee joint medial side to cut the skin and muscle, and the deep tissue was separated by a bland method to expose the femoral head medial side. Then, the periosteum was removed with a periosteum dissection device to fully expose the visual field. The rotary speed of the implant was adjusted to $1000 \mathrm{rpm}$. Drilling was carried out at a slow speed on the vertical bone surface inside the femoral head, and the drilling process was cooled with sterile normal saline. Bone fragments were removed by washing, and the holes were gradually expanded at a constant speed to prepare for hole formation ( $6 \mathrm{~mm}$ depth and $4 \mathrm{~mm}$ diameter). Finally, the metal implant material was placed into the hole so that its top was flush with the bone surface. The wound was repeatedly washed with normal saline, and the periosteum, muscle, and skin were stratified and sutured (Fig. 2).

Postoperatively, $40 \mathrm{mg} / \mathrm{kg}$ ceftiafecan was injected intraperitoneally to prevent wound infection. When the rabbits woke up and resumed exercise, they were sent back to the feeding center. They were fed normally in a single cage, and their affected limbs were allowed to move freely with weight. Within 5 days after the operation, the rabbits were daily injected with $40 \mathrm{mg} / \mathrm{kg}$ antibiotics into their buttocks to prevent infection, and the wounds were daily checked to ensure cleanliness. According to the experimental design, animals were killed by air embolization at the 12th and 16th weeks after surgery. The femoral end with the implant was dissected, and the bone segment with the implant material, as well as the organs (liver, kidney, and heart) was removed and quickly stored in $4 \%$ neutral buffer formalin solution for subsequent experiments.

\subsubsection{Gross sample observation}

Animals were killed according to the predetermined time point of the experimental design, and anatomical materials were collected immediately. During the anatomical surgery process, incision healing 
at the implantation site, the soft tissue around the implant, and the combination of the implant and bone tissue were observed. The femur with the implant material was removed, labeled, numbered, and photographed.

\subsubsection{Imaging evaluation}

The femur's anterior and lateral films (12 and $16 \mathrm{w}$ ) with implant materials were taken by the X-ray machine (Third Xiangya Hospital of Central South University) to observe the interface between the implant and the surrounding bone tissue. The radiographs were observed and analyzed to check for the presence of low-density images and to observe new bone formation around the implant material. If the low-density area was greater than $0.5 \mathrm{~mm}$, it indicated bone tissue absorption around the implant.

\subsubsection{Hard tissue observation by grinding and staining}

The femur with the implant material was immersed in a $4 \%$ neutral buffer formalin solution. After $48 \mathrm{~h}$, the femur was rinsed with water, dehydrated in a graded ethanol series $(70 \%, 80 \%, 90 \%$, and $95 \%)$, and soaked in different concentrations of Technovit7200 resin solution (the solvent was anhydrous ethanol). Embedding polymerization was performed in a light-curing machine. The tissue mass was removed and sliced along the implant material long axis using a German Exakt-300CP hard tissue slicer. The tissue was cut into $200 \mu \mathrm{m}$ thick slices and grounded to $25 \mu \mathrm{m}$ with 300\#, 800\#, 1200\#, and 2000\# sandpaper using a German Exakt400S grinding machine. Cut marks were removed by polishing with 4000 mesh sandpaper. Non-decalcified slices ( $20 \mu \mathrm{m}$ thick) were retained and pasted on $0.2 \mathrm{~cm}$ glass slides. The slices were stained with $1 \%$ methylene blue solution for $10 \mathrm{~min}$, washed with deionized water to remove dye excess, stained with $2 \%$ alkaline magenta for $5 \mathrm{~min}$, washed with $95 \%$ and $100 \%$ alcohol for $10 \mathrm{~min}$ each, treated with xylene transparent, and finally sealed. The section's tissue morphology was observed under a biological microscope.

\subsection{Statistical analysis}

Statistical analysis was performed using GraphPad Prism 6 software and two-way analysis of variance (ANOVA). A $p<0.05$ was considered statistically significant.

\section{Results}

\subsection{Ti-45Zr alloy blood compatibility}

Biomaterial blood compatibility is one of the most important criteria to determine whether the material can be successfully applied in clinical practice. If the implant material's blood compatibility is poor, a series of reactions such as hemolysis, coagulation, and immune rejection will occur in the body, leading to implant failure. Therefore, a hemolysis test was used to evaluate the Ti-45Zr alloy blood compatibility. The hemolysis rate was calculated according to Eq. 1-1 and results are shown in Table 1. The Ti-45Zr alloy hemolysis rate was $1.18 \%$ and followed national standards (biomaterials hemolysis rate $<5 \%$ ). Therefore, the Ti-45Zr alloy had low hemolysis and good blood compatibility. 
Hemolysis ratio $=[($ Experimental group OD - Negative control group OD $) /($ Positive control group OD Negative control group OD)] * $100 \%(1-1)$

Table 1

Ti-45Zr alloys hemolysis rate

\begin{tabular}{|c|c|c|c|c|c|}
\hline \multirow[t]{2}{*}{ group } & \multicolumn{3}{|c|}{ OD value } & \multirow[t]{2}{*}{ mean value } & \multirow[t]{2}{*}{ hemolysis ratio } \\
\hline & 1 & 2 & 3 & & \\
\hline $\mathrm{Ti}-45 \mathrm{Zr}$ & 0.038 & 0.035 & 0.032 & 0.038 & $1.18 \%$ \\
\hline Negative & 0.024 & 0.025 & 0.025 & 0.0243 & \\
\hline Positive & 0.942 & 0.913 & 0.866 & 0.907 & \\
\hline
\end{tabular}

\subsection{In vivo osteo-immunomodulation \\ 3.2.1. Inflammatory factors secretion}

Macrophages are the main inflammatory immunomodulatory cells, which regulate and terminate the inflammatory response by secreting a variety of inflammatory factors. IL- 6 and IL-10 are major proinflammatory and anti-inflammatory factors in this response, playing an important role in its process and outcome. In this study, the IL- 6 and IL-10 in the rats' blood were detected by ELISA at different time points (Fig. 3). In general, IL-6 concentrations reached the highest level on the 9th day and were significantly downregulated on the 14th day. The IL-10 concentration in the Ti-45Zr group reached the highest level on the 9 th day, and on the 14 th day was downregulated.

\subsubsection{Inflammatory response after implantation}

Implantation can trigger the body's immune defense mechanism. The most typical response is implanting inflammation. The inflammatory response outcome regarding the implant stability and bone formation ability is very important. In this study, the inflammatory response around the implant material was observed by HE staining. On the 3rd day, the main pathological change in each group was inflammatory exudation (Fig. 4). Inflammatory cells were mainly neutrophils and a small number of lymphocytes and monocytes was observed. No clear pathological changes were observed in the bone tissue around the cave implantation. On the 9th day, There were a few osteoid matrices and osteoblasts in the inflammatory foci. The inflammatory cells were mainly monocytes and macrophages. The number of macrophages increased compared with the 3rd day, and a few multinucleated macrophages were observed. Around the 14th day, fibrous connective tissue hyperplasia formed inside the implantation hole. Interstitial mononuclear cell infiltration was visible in the trabecular bone structure, bone matrix, cartilage cells, and osteoblasts. New bone formation occurred in the later inflammatory response stage and the inflammation prognosis was good.

\subsubsection{Macrophages' infiltration}


Macrophages are the first cells that contact the material and participate in immune regulation. In the inflammatory response stage, macrophages will arrive at the material's surface mediated by chemokines and participate in the inflammatory response regulation process. Early macrophages' infiltration contributes to the initial inflammatory response and debridement at the implant site.

Immunohistochemical staining was used to mark the specific marker CD68 expression on the surface of macrophages, allowing the evaluation of macrophages' infiltration distribution around the implanted material. Macrophages with brown nuclei were deeply stained, and the red arrow indicates the CD68 positive staining area (Fig. 5). On the 9th day, the positive staining area increased compared to the 3rd and 14th days. Also on the 9th day, macrophages' infiltration around the implanted hole increased, consistent with HE staining results. The staining area optical density was measured by Image-Pro Plus 6.0 Image analysis system, and the average for each group was calculated. On the 9th day, CD68 positive region average optical density was significantly increased compared to the 3rd day. On the 14th day, the average CD68 positive region optical density decreased (Fig. 6).

\subsubsection{Macrophages polarization}

Macrophage polarization exists in a wide range from the "classically activated" M1 type to the "bypassactivated" M2 type. M1 and M2 are known as two representative and typical phenotypes, that can secrete a variety of cytokines in different tissue microenvironments. M1-type macrophages mainly secrete proinflammatory factors, while M2-type mainly secrete anti-inflammatory ones. The two polarization phenotypes markers are different, such as tumor necrosis factor-a (TNF-a) and iNOS for M1 phenotypes, and Arg1, interleukin-1 ra (IL-1 ra) and differentiated antigen cluster-206 (CD206) for M2 type cells.

In this study, INOS2 and Arg1 markers on the surface of M1 and M2 macrophages, respectively, were labeled by immunohistochemical staining to evaluate macrophages' polarization. Image-Pro Plus 6.0 Image analysis system was used to analyze the immunohistochemical staining images, and the ratio of iNOS2 ${ }^{+}$cells and $\mathrm{Arg}^{+}$cells in the number of $\mathrm{CD} 68^{+}$cells was calculated, the $\mathrm{M} 1$ and $\mathrm{M} 2$ polarization conversion of macrophages was evaluated (Fig. 7). On the third day, at the inflammation initial stage, the proportion of M1 macrophages was relatively high, but with time extension, it gradually decreased (Fig. 7a). However, the proportion of M2 macrophages was the lowest on the 3rd day and reached the highest on the 9th day, followed by a slight downregulation (Fig. 7b). On the 14th day, M1 and M2 macrophages showed moderate expression, and the polarization ratio of M1/M2 tended to be balanced. These results indicated that the Ti-45Zr group had a positive effect on the polarization phenotype.

\subsection{In vivo osseointegration}

\subsubsection{Ti-45Zr alloy systemic toxicity evaluation}

At week 16, animals were sacrificed, immediately dissected, and the main organs (liver, heart, and kidney) were removed, placed in $4 \%$ neutral buffer formalin solution, fixed for $48 \mathrm{~h}$, dehydrated, and embedded. Pathological sections were stained with HE (Fig. 8). No pathological changes (ex. deformation, necrosis, 
or atrophy) were observed in the animals' main organs (liver, heart, and kidney). The tissue cell morphology was normal, and no inflammatory or apoptotic cells were observed, indicating that the Ti$45 \mathrm{Zr}$ alloy did not release toxic ions in vivo. Therefore, no clear toxic or side effects on the animals' main organs were observed.

\subsubsection{Gross sample observation}

At the 12th and 16th weeks, animals were sacrificed for dissection and bone segments with implant materials were removed. The implantation site wound healing in each group was good, and the muscle color was normal and elastic, and there was no redness, no inflammatory exudate, and no scar tissue proliferation. The muscle was gradually stripped and the femur was fully exposed. The soft tissues of the four groups were normal, without inflammatory secretions. The implanted materials were tightly embedded in the bone tissue without loosening or propulsion, and no bone resorption or destruction was observed with the naked eye (Fig. 9).

\subsubsection{X-ray evaluation}

X-ray examination of the femur with the implant was performed. Femur's anteroposterior and lateral radiographs (12 and 16 weeks) are shown in Fig. 10. At the 12th week after surgery, X-ray films showed that there was no bone destruction or absorption around the Ti-45Zr alloy. On the 16th postoperative day, there was no obvious low-density dark area around the implant, while white bone lines could be seen in some areas, indicating increased bone density. The implant material was closely combined with the surrounding bone tissue.

\subsubsection{Hard tissue slice histomorphological analysis}

The bone tissue with the implant was sectioned and stained to observe the interface between the material and the bone tissue. After 12 weeks of healing, new bone was formed around all implants, while a small amount of osteoid could be seen at the bone-implant interface without significant fibrous tissue (Fig. 11). Sixteen weeks later, the amount of new bone formed around the implant increased. The new bone was in close and direct contact with the implant surface, and blue osteoblasts could be seen in a few gaps.

\section{Discussion}

The hemolysis test results confirmed that Ti-45Zr alloy has good blood compatibility and no body side effects, consistent with the systemic short-term toxicity test results. In the systemic toxicity test, no toxic reactions, no abnormalities in the important organs, and no obvious pathological changes in tissue sections were observed in the animals. This indicates that the Ti-45Zr alloy does not have a systemic toxic reaction, confirming that $\mathrm{Zr}$ and $\mathrm{Ti}$ are non-toxic metal elements, and both $\mathrm{Zr}$ and $\mathrm{Ti}$ are life-reactive metals with high biosecurity. 
Further, the Ti-45Zr alloy immune reaction in vivo was analyzed. The immune system is closely related to the skeletal system, and they share many cytokines, receptors, signaling molecules, and transcription factors [3]. Immune cells play a key role in bone homeostasis. Dental implants are implanted into bone tissue through invasive surgery. As a foreign body, the implant is recognized by the immune system and triggers a significant immune response that affects bone cell behavior. The immune response outcome will ultimately determine the fate of the implanted material in vivo [4]. Therefore, the immune response is a key factor to evaluate bone-implant materials stability. The immune response effects on bone integration ability of bone biomaterials is a "double-edged sword". A favorable immune response can produce a local microenvironment that promotes osteogenesis, while an inappropriate one can lead to chronic inflammation and fibrous encapsulation around the implant. Macrophages' polarization and their anti-inflammatory and pro-inflammatory properties play an important role in host response after material implantation and have a significant impact on the outcome and osteosseointegration long-term stability [5].

In this study, HE staining and serum biochemical experiments were used to analyze the Ti-45Zr alloy inflammatory response after implantation in vivo. On the 3rd day after implantation, the body was in an acute inflammation period, with mainly neutrophils and lymphocytes and mononuclear cells infiltration (HE dyeing - Fig. 4). Around implanted holes, the macrophage infiltration was low in the beginning but with time extension the number of macrophages increased gradually and had a tendency to migrate to the material surface. On the 9th day, the number of macrophages was significantly higher than on day 3 , indicating that the organism's immune response enhanced with time. On the 14th day, there was new bone formation around all the implant holes, indicating that the inflammation was progressing well. Inflammation-related factors in serum also increased with time. On the 3rd day, IL-6 was significantly higher than IL-10. On the 9th day, both IL-6 and IL-10 were highly expressed, while downregulated on day 14 , following the normal course of inflammation. IL- 6 is a multifunctional proinflammatory cytokine secreted by $\mathrm{M} 1$ macrophages, involved in bone metabolism inflammation and regulation. Therefore, it plays an important role at the bone-material interface. IL- 6 can induce RANKL expression and indirectly promote osteoclast generation and activation by RANKL/RANK OPG system. Additionally, IL-6 is essential for bone tissue healing early stages, and loss of IL-6 delays bone tissue mineralization and remodeling [6, 7]. IL-10 is an inflammatory cytokine mainly produced by activated M2 macrophages and to a lesser extent by T cells. IL-10 supports BMSCs homing, proliferation, and osteogenic differentiation [8]. It can also inhibit the release of a variety of inflammatory factors and has the potential to inhibit antigen presentation. Therefore, IL-10 inhibits immune enhancement and inflammation. Recent studies have shown that IL-10 can inhibit osteoclasts formation [9], thereby inhibiting bone destruction and resorption occurrence. Altogether, moderate expression of IL- 6 and IL-10, during the inflammatory phase, is beneficial to new bone formation.

Immune cells play a key role in bone homeostasis. This can be achieved through the release of cytokines that regulate osteogenesis, inducing, or inhibiting, bone formation. Macrophages are crucial to efficient osteoblast differentiation, and, in vivo, macrophage depletion results in complete loss of osteoblastmediated bone formation. The effect of implantable materials on macrophages' polarization phenotype 
directly determines the osteointegration effect and long-term stability. Immunohistochemical staining results reflected the macrophage's transformation phenotype (Fig. 5 and Fig. 6). On day 3, M1 macrophages are the majority around the implant material, and on day 9, the M2 macrophage polarization ratio increases, indicating a macrophage polarization phenotype transformation. Spiller pointed out that macrophages can have a short span of 3 days when M2 macrophages were completely differentiated into M1 macrophages and vice versa [10], consistent with this experiment's results. On the 14th day, M1 and M2 macrophages polarization ratio was downregulated. M1 macrophages may play a key role in osteogenesis early and middle stages, while M2 macrophages may contribute to matrix late mineralization. Moderate conversion from M1 to M2 phenotype may be critical for implant materials fracture healing and osteointegration [11]. A moderate transition from the pro-inflammatory phenotype M1 to the immunoregulatory, or anti-inflammatory, phenotype M2 can be an important aspect in the bone healing process promotion, leading to a functional recovery rather than scar tissue formation [12-14]. On the 3rd day, the inflammation early stage had mainly the M1 type (high proportion), while on the 14th day, the M2 type was dominant (moderate proportion) (Fig. 8). This indicates that the Ti-45Zr alloy conduced the macrophages' polarization balance, new bone formation, and long-term stability maintenance.

New Zealand white rabbits were used as animal models, and bone integration stability and ability were through in vivo implantation experiments. Satisfactory osseointegration between the host bone and the implant material is crucial for dental implants' long-term stability. After 12 weeks of healing, new bone was formed around the implant, while a small amount of osteoid could be seen at the bone-implant interface without clear fibrous tissue (Fig. 11). There was a certain gap between the bone tissue and the implant interface, in which osteoblasts could be seen. With implantation time prolongation, new bone formation around all implants increased until week 16, and the space between the new bone and the implant material surface decreased, leading to close and direct contact. There were no blank non-contact images and low-density bone absorption images between Ti-45Zr alloy and bone tissue (X-ray films Fig. 10). The results showed that the Ti-45Zr alloy had direct contact with bone tissue, and there was no obvious fibrous layer formed between the alloy and bone tissue. The implant material osteointegration ability is related to many factors, including alloying elements, surface properties, elastic modulus, porosity, and pore size. In this study, the Ti-45Zr alloy's good osseointegration ability may be attributed to its excellent mechanical properties (low elastic modulus). Additionally, zirconium ( $\mathrm{Zr}$ ) and titanium ( $\mathrm{Ti}$ ) are metals with good osseointegration activity. After 6 months of $\mathrm{Zr}$ and $\mathrm{Ti}$ threaded implantation into pigs' oral alveolar bone, Kulakov et al. confirmed that the amount of new bone formation around $\mathrm{Zr}$ implants was higher than that around Ti implants under the same conditions [15]. The success and periimplant bone absorption rates of the Ti-Zr narrow-diameter implant were comparable to that of $\mathrm{cpTi}$ implants of the same size [16].

Overall, the Ti-45Zr alloy has superior biocompatibility and it was conducive to macrophages' polarization balance. Particularly, Ti-45Zr showed good osteogenic activity in vivo, being a promising alternative to cpTi as a dental implant. However, this in vivo study did not provide a quantitative analysis of bone formation around the implant, which requires further investigation. 


\section{Conclusion}

The Ti-45Zr alloy showed good blood compatibility, had no body's toxic side effects and responded to the inflammatory response in vivo. With implantation time extension, the inflammation recovery was satisfactory and new bone formation was observed around the material. Also, the Ti-45Zr alloy was beneficial to macrophages' polarization balance. However, in vivo analyses demonstrated that the Ti-45Zr had good osteogenic activity. Altogether, these results suggested that the Ti-45Zr showed excellent biocompatibility, immunoregulatory ability, and osteointegration to support bone formation, being a prospect for dental implant materials transformation and application.

\section{Declarations}

\section{Availability of data and materials}

The data and materials used in the current study are all available from the corresponding author upon reasonable request.

\section{Conflicts of interest}

The authors declare no conflict of interest.

\section{Acknowledgments}

Not applicable.

\section{Funding}

This work was supported by the Natural Science Foundation of Hunan Province (No. 2020JJ5862).

\section{Author information}

Affiliations

State Key Laboratory of Powder Metallurgy, Central South University, Changsha 410083, PR China

Pinghua Ou, Taomei Zhang, Jianying Wang, Jianming Ruan

Department of Stomatology, The Third Xiangya Hospital, Central South University, Changsha 410013, PR China

Pinghua Ou, Cui Li, Chunsheng Shao,

Contributions 
Jianming Ruan and Pinghua Ou conceived and designed the experiments; Pinghua Ou and Taomei Zhang performed the experiments; Jianying Wang contributed reagents/materials; Cui Li and Chunsheng Shao contributed data analysed; Pinghua Ou wrote the manuscript. All authors read and approved the final manuscript.

Corresponding authors

Correspondence to Jianming Ruan.

\section{Ethics declarations}

Ethics approval and consent to participate

The experiments involving animals were approved by the animal care and use ethical committee of Central South University (No: 2019syuw0225) and Hunan Academy of Traditional Chinese Medicine (No: 2021-0023), complied with the Guide for the Care and Use of Laboratory Animals approved by the National Institutes of Health.

Consent for publication

Not applicable.

Competing interests

The authors declare that there are no competing interests.

\section{References}

[1] Okazaki Y, Rao S, Ito Y, et al. Corrosion resistance, mechanical properties, corrosion fatigue strength and cytocompatibility of new Ti alloys without Al and V [J]. Biomaterials, 1998, 19(13):1197-1215.

[2]Ou P H, Hao C, Liu J, He R G, et al. Cytocompatibility of Ti-xZr alloys as dental implant materials [J]. Journal of Materials Science: Materials in Medicine, 2021; 32(5):50. DOI:10.1007/s10856-021-06522-w.

[3] Albrektsson T, Chrcanovic B, Jacobsson M, et al. Osseointegration of implants-a biological and clinical overview [J]. JSM Dental Surgery, 2017, 2:1022-1027.

[4] Xu X C, Lin Z H, Dong W. The effects of Titanium surfaces modified with an antimicrobial peptide GL13K by silanization on polarization, anti-inflammatory, and proinflammatory properties of macrophages [J]. BioMed Research International, 2020, 2327034 
[5] Mengchi X, Dong Z, Jiang C, et al. In vitro assessment of three-

dimensionally plotted nagelschmidtite bioceramic scaffolds with varied

macropore morphologies [J]. Biomaterials, 2014, 10:463-476.

[6] Yang X, Ricciardi B F, Hernandez-Soria A, et al. Callus mineralization and maturation are delayed during fracture healing in interleukin-6 knockout mice [J]. Bone, 2007, 41(6):928-936.

[7] Bryan N B, Buddy D R, Stuart B G, et al. Macrophage polarization: An opportunity for improved outcomes in biomaterials and regenerative medicine [J]. Biomaterials, 2012, 33(15):3792-3802.

[8] Klopfleisch R. Macrophage reaction against biomaterials in the mouse model-Phenotypes, functions and markers [J]. Acta Biomaterialia, 2016, 43:3-13.

[9] Mohamed S G K, Sugiyama E, Shinoda K, et al. Interleukin-10 inhibits RANKL-mediated expression of NFATcl in part via suppression of c-Fos and c-Jun in RAW264.7 cells and mouse bone marrow cells [J]. Bone. 2007, 41(4):592-602.

[10] Spiller KL, Nassiri S, Witherel CE, Anfang RR, Ng J, Nakazawa KR, Yu T, Vunjak-Novakovic G. Sequential delivery of immunomodulatory cytokines to facilitate the M1-to-M2 transition of macrophages and enhance vascularization of bone scaffolds. Biomaterials, 2015;37:194-207.

[11] Kara L S, Sina N, Claire E W, et al. Sequential delivery of immunomodulatory cytokines to facilitate the M1-to-M2 transition of macrophages and enhance vascularization of bone scaffolds [J]. Biomaterials, 2015, 37:194-207.

[12] Brown B N, Londono R, Tottey S, et al. Macrophage phenotype as a predictor of constructive remodeling following the implantation of biologically derived surgical mesh materials [J]. Acta Biomaterialia, 2012, 8(3):978-987.

[13] Sussman E M, Halpin M C, Muster J, et al. Porous implants modulate healing and induce shifts in local macrophage polarization in the foreign body reaction [J]. Annals of Biomedical Engineering, 2014, 42(7):1508-1516.

[14] Anderson J M, Jones J A . Phenotypic dichotomies in the foreign body reaction [J]. Biomaterials, 2007, 28(34):5114-5120.

[15] Kulakov O B, Doktorov A A, Diakova S V, et al. Experimental study of osseointegration of zirconium and titanium dental implants [J]. Morfologiya, 2005, 127(1):52-55.

[16] legami C M, Uehara P N, Sesma N, et al. Survival rate of Ti-Zr narrow diameter dental implants versus commercially pure diameter dental implants versus commercially pure titanium diameter dental implants [J]. Clinical Implant Dentistry and Related Research, 2017, 19(6):1015-1022. 
Figures
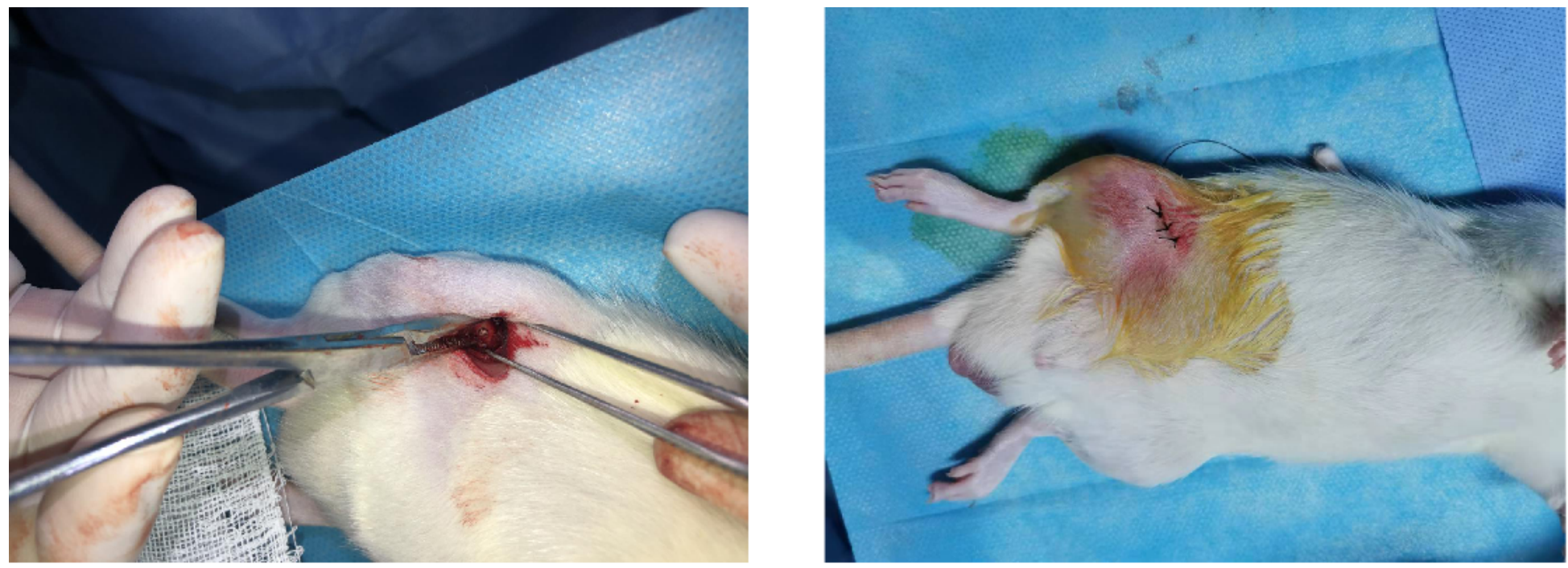

Figure 1

Rats implantation surgery process diagram.
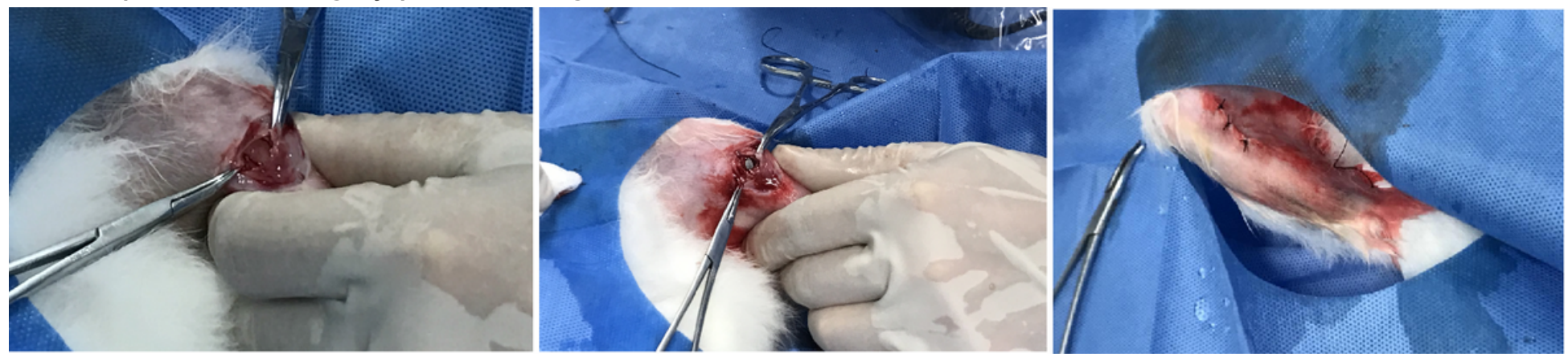

Figure 2

Rabbits implantation surgery process diagram.

(a)

(b)
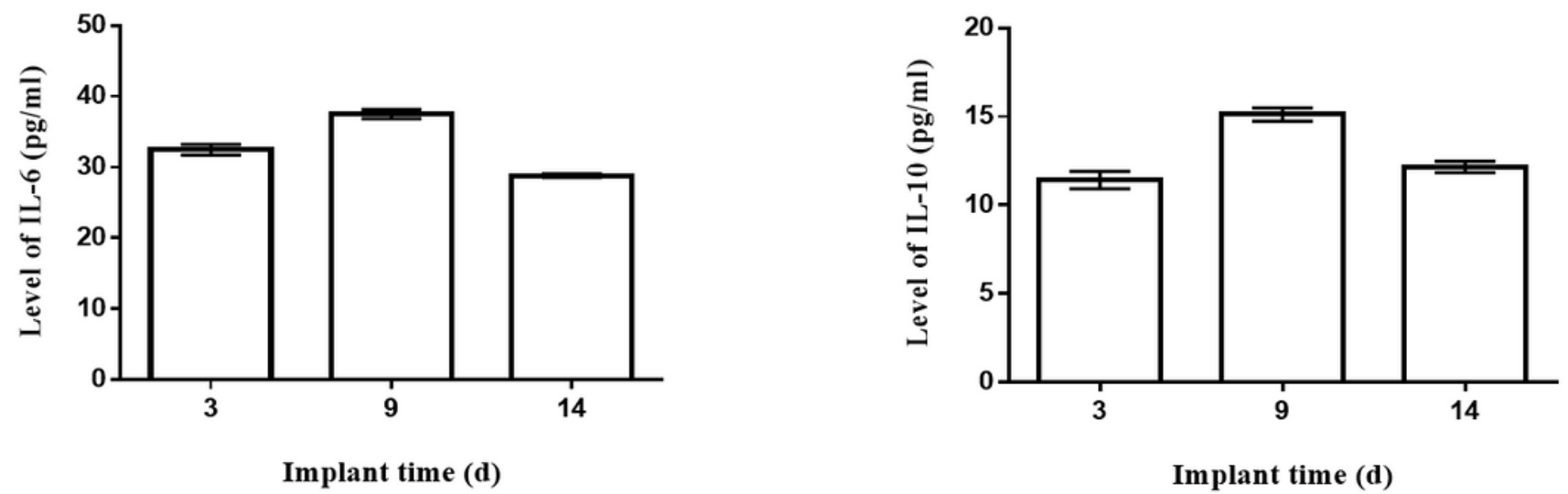

Figure 3 
Serum concentrations of inflammation-related factors: (a) IL-6; (b) IL-10. ${ }^{\star} p<0.05$; ${ }^{\star \star} p<0.01$; $* \star \star p<$ 0.001 .

$3 \mathrm{~d}$

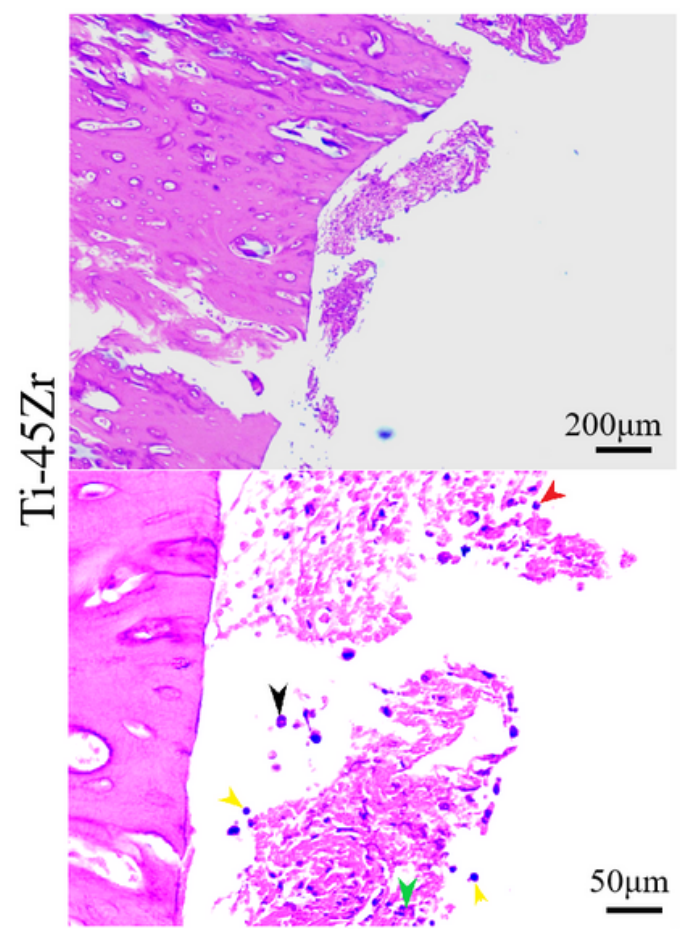

$9 \mathrm{~d}$

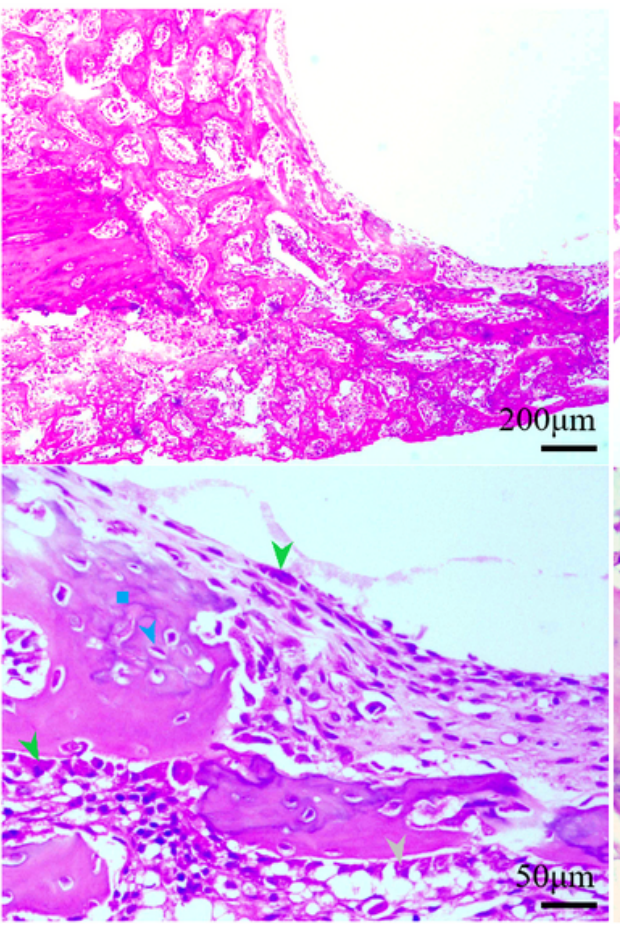

$14 \mathrm{~d}$

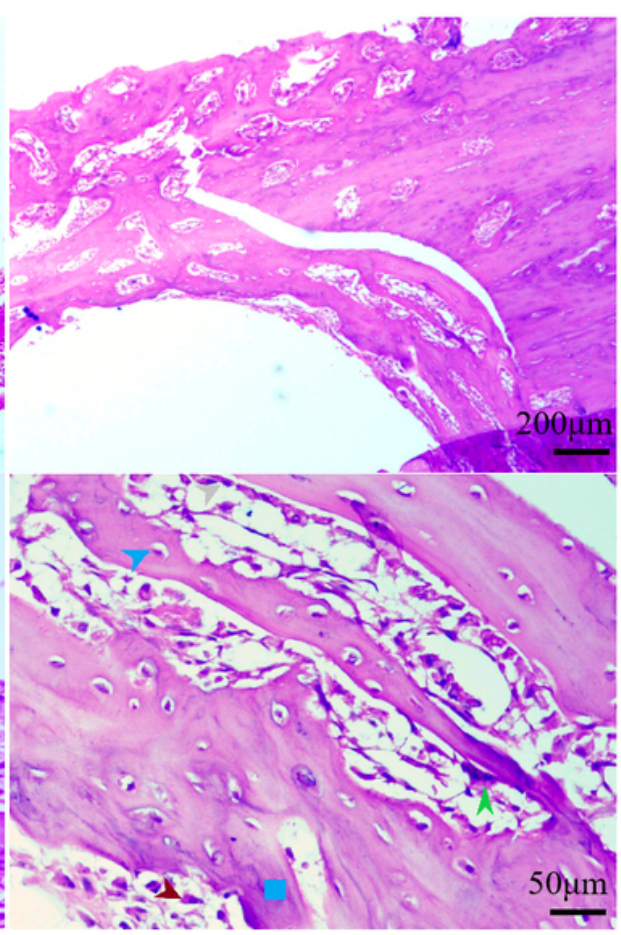

\section{Figure 4}

HE staining histological observation: neutrophils (red arrows); macrophages (green arrows); lymphocytes (yellow arrows); monocytes (black arrows); osteoblasts (gray arrows); chondrocytes (blue arrows); bone like matrix (blue square).

\section{$3 \mathrm{~d}$}

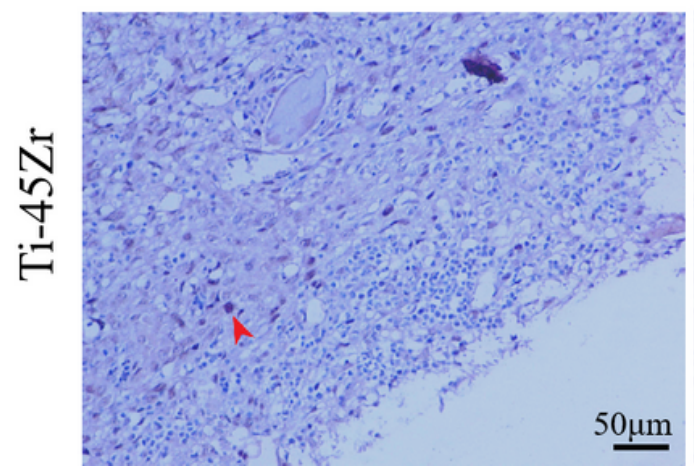

$9 \mathrm{~d}$

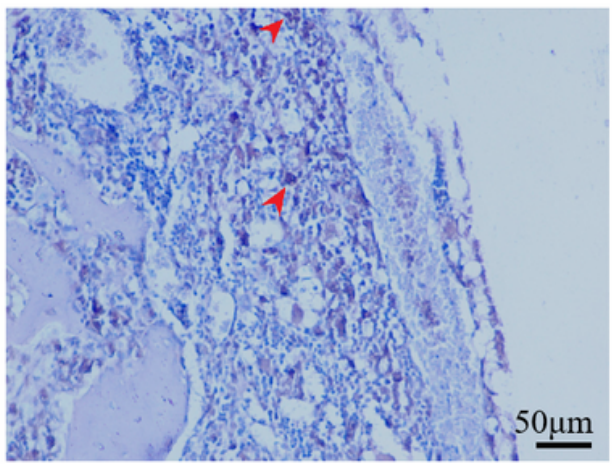

$14 \mathrm{~d}$

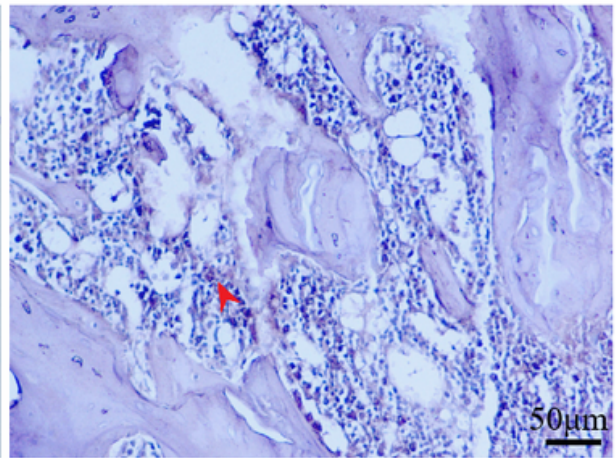

\section{Figure 5}

CD68 immunohistochemical staining and histological observation at 3, 9, and 14 days after Ti-45Zr alloy implantation (red arrow indicates positive color area). 


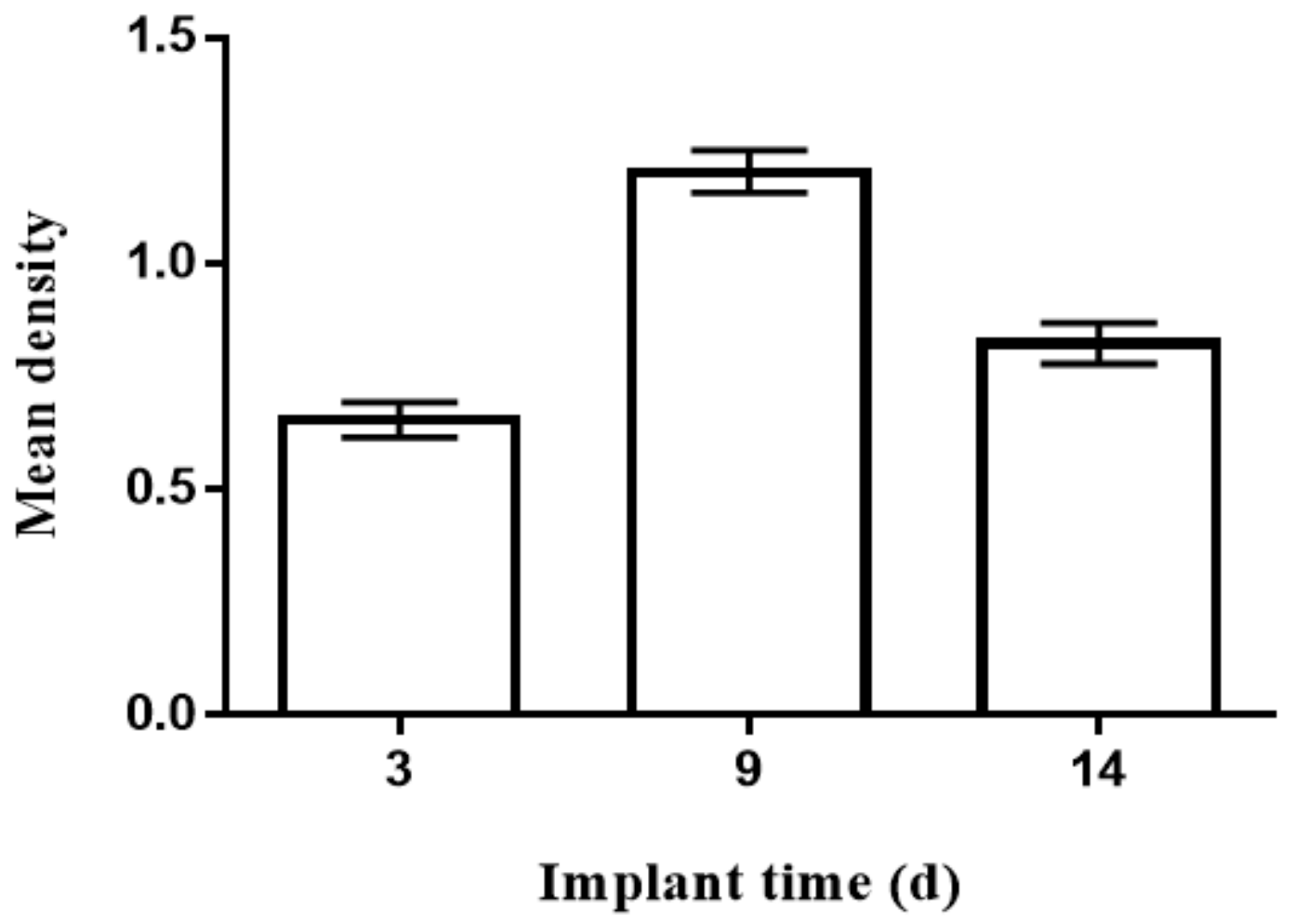

Figure 6

CD68 positive fraction mean density at 3,9 , and 14 days after implantation. ${ }^{*} p<0.05$; $* \star p<0.01$; $* \star * p<$ 0.001 .

(a)
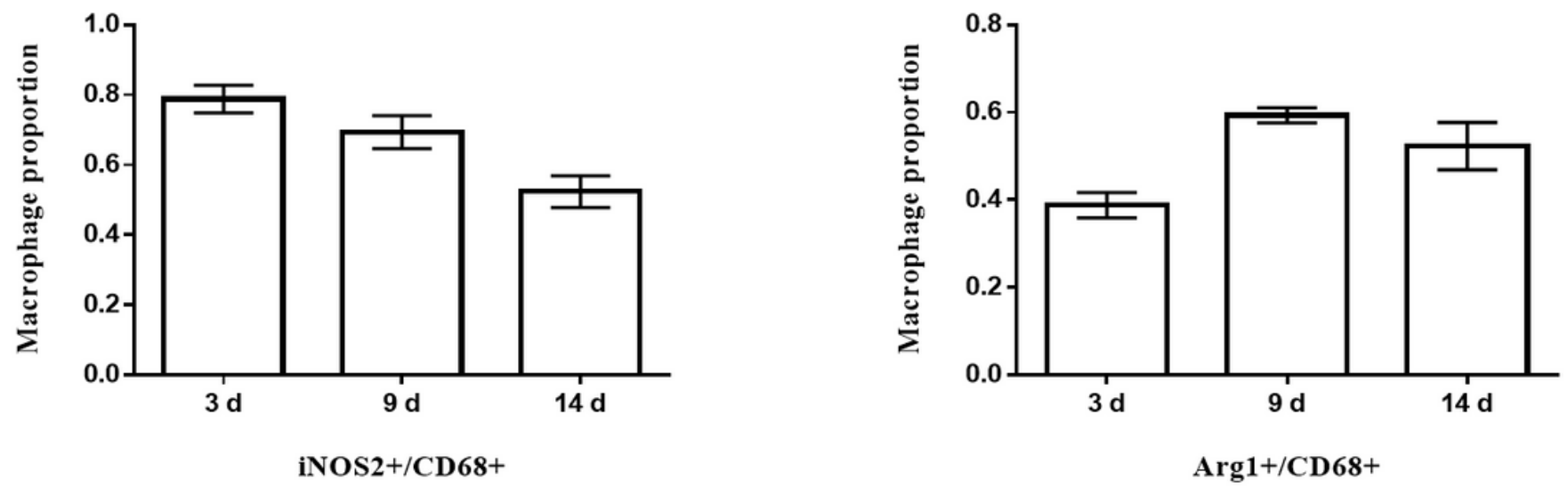

Figure 7

iNOS2+ and Arg1+ cells proportion in CD68+ cells at 3, 9, and 14 days after implantation. 


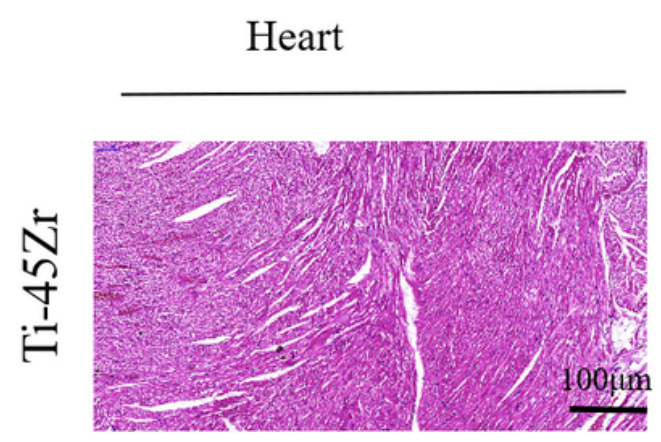

Liver

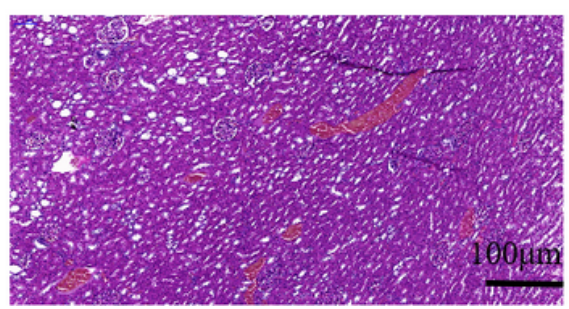

Kidney

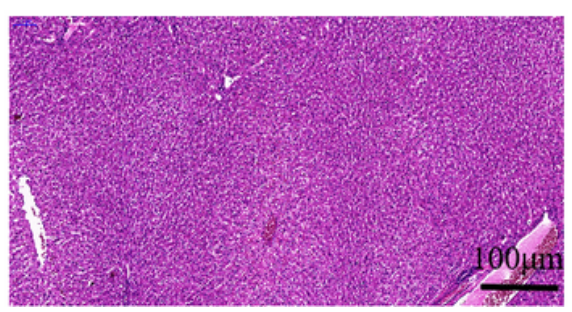

\section{Figure 8}

Heart, liver, and kidney's HE staining.

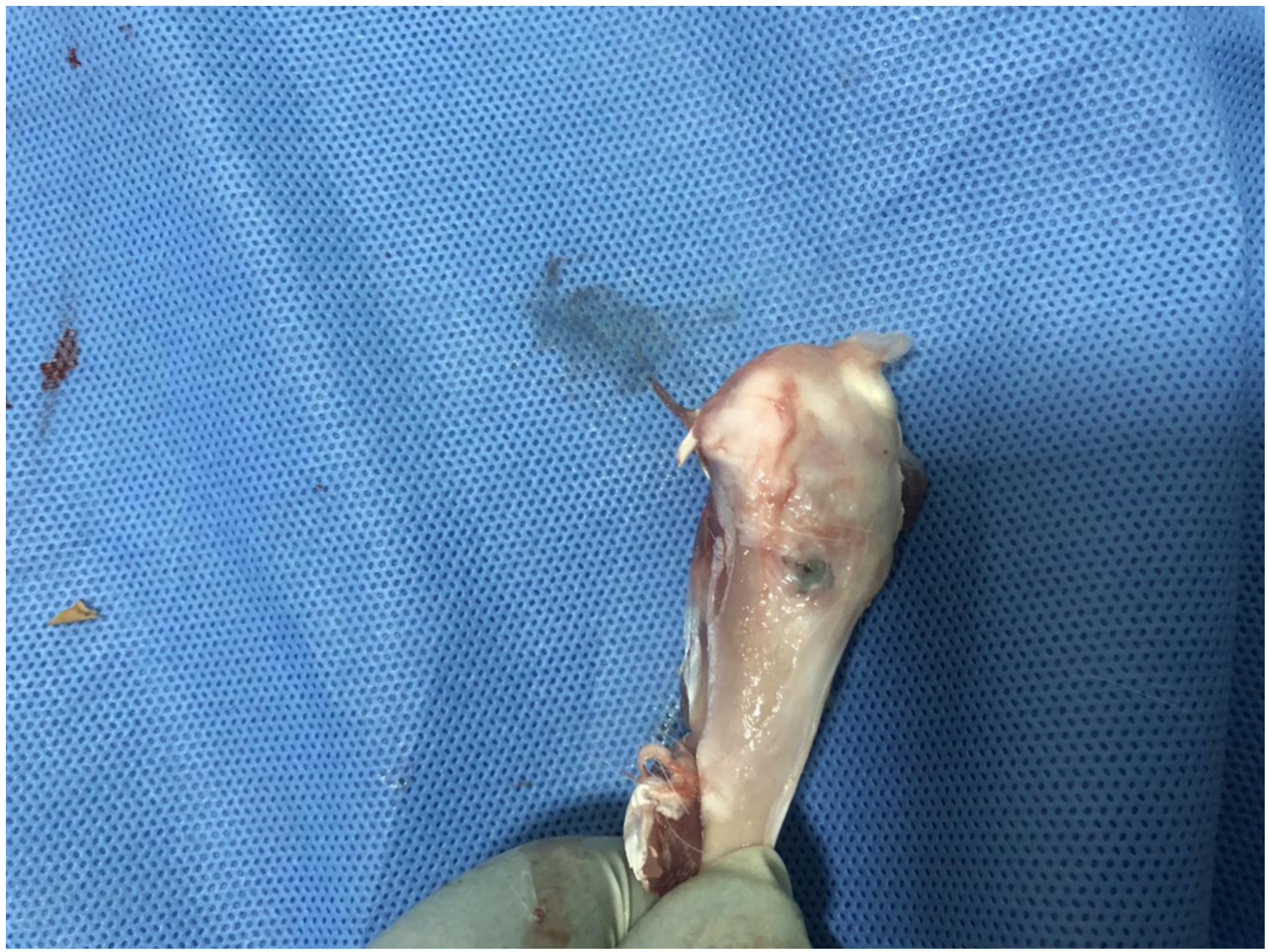

Figure 9

A femoral specimen with implant material removed postoperatively. 

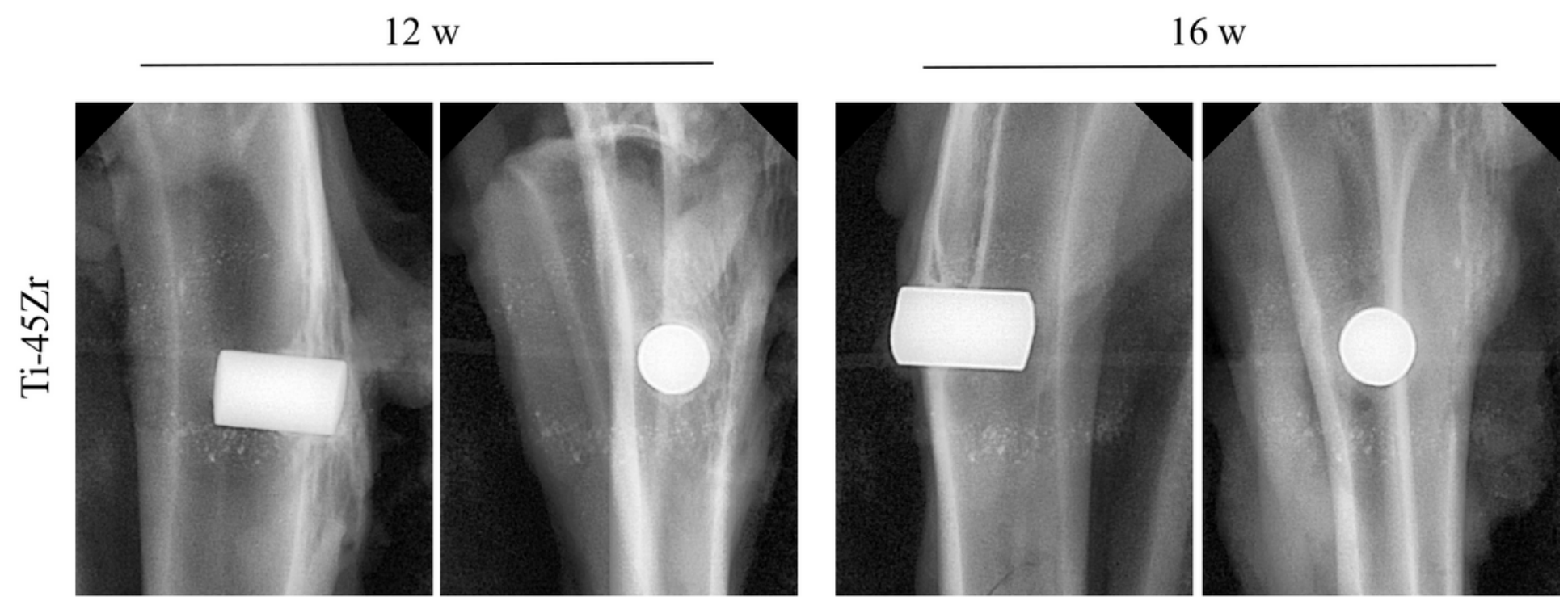

Figure 10

Anteroposterior and lateral radiographs of rabbit femoral specimens with implant material.
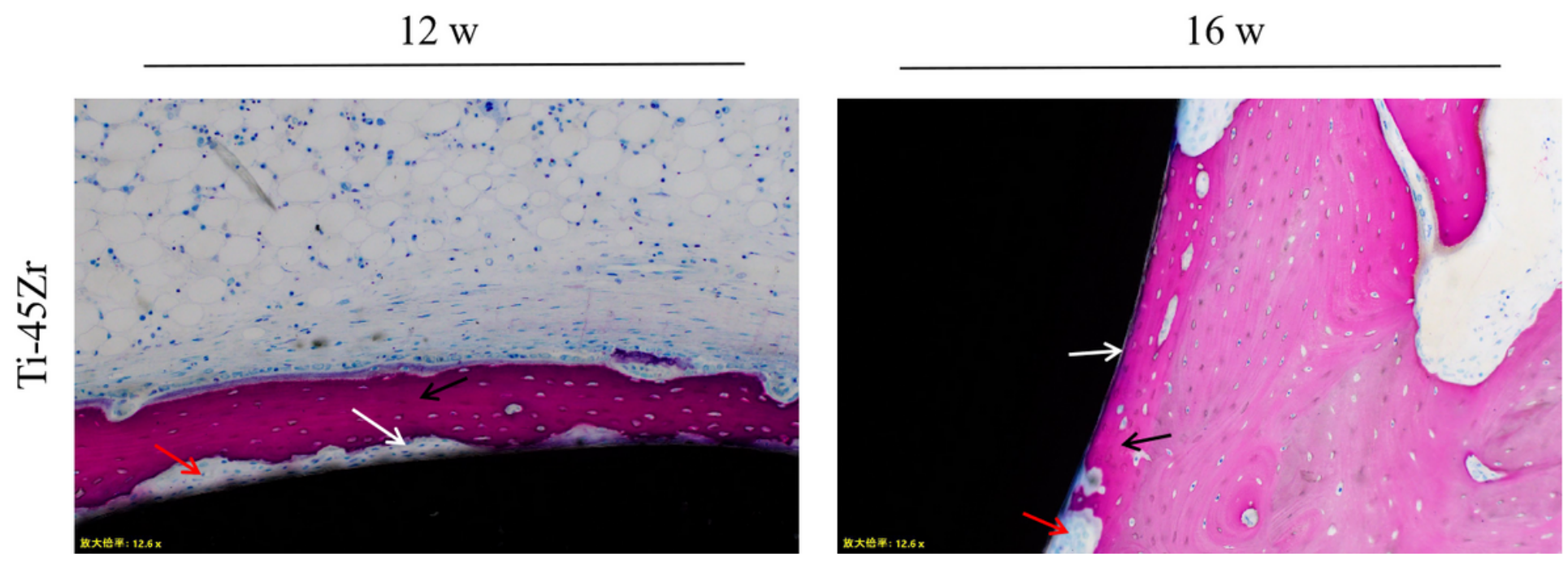

\section{Figure 11}

Histological signs of bone formation around the implant surface stained with methylene blue-basic magenta: bone-binding interface (white arrow); osteoblast (red arrow); new bone tissue (black arrow). 